\title{
Effect of glucopyranosylidene-spiro-thiohydantoin on glycogen metabolism in liver tissues of streptozotocin-induced and obese diabetic rats
}

\author{
TIBOR DOCSA ${ }^{1}$, KATALIN CZIFRÁK ${ }^{2}$, CSABA HÜSE $^{2}$, LÁSZLÓ SOMSÁK ${ }^{2}$ and PÁL GERGELY ${ }^{1}$ \\ ${ }^{1}$ Cell Biology and Signalling Research Group of the Hungarian Academy of Sciences, \\ Department of Medical Chemistry, Research Centre for Molecular Medicine; \\ ${ }^{2}$ Department of Organic Chemistry, Faculty of Science, University of Debrecen, H-4032 Debrecen, Hungary
}

Received December 9, 2010; Accepted March 21, 2011

DOI: $10.3892 / \mathrm{mmr} .2011 .464$

\begin{abstract}
The major role of liver glycogen is to supply glucose to the circulation in order to maintain normal blood glucose levels. In the muscle and liver, the accumulation and breakdown of glycogen are regulated by the reciprocal activities of glycogen phosphorylase and glycogen synthase. Glycogen phosphorylase catalyses the key step of glycogen degradation and its activity is inhibited by glucose and its analogues. Thus, any readily accessible inhibitor of glycogen phosphorylase may serve as a potential therapy for non-insulin-dependent or type 2 diabetes. Hepatic glycogen phosphorylase has been identified as a novel target for drugs that control blood glucose concentration. Glucopyranosylidene-spiro-thiohydantoin (TH) was found to be one of the most potent glucose derivates, inhibiting the catalytic activity of both muscle and liver glycogen phosphorylase. Here, we demonstrated the co-ordinated regulation of glycogen phosphorylase and synthase by $50 \mu \mathrm{M} \mathrm{TH}$ in liver extracts of Wistar rats, resulting in the activation of synthase by a shortening of the latency compared to control animals. TH was also effective in lowering blood glucose levels and restoring hepatic glycogen content in streptozotocininduced diabetic rats. Furthermore, intravenous administration of $\mathrm{TH}$ to Zucker diabetic fatty rats significantly decreased hepatic glycogen phosphorylase $a$ levels, and the activation of synthase was initiated without any delay.
\end{abstract}

\section{Introduction}

Type 2 diabetes (non-insulin-dependent diabetes mellitus) is a major public health problem with severe complications, such

Correspondence to: Dr Pál Gergely, Department of Medical Chemistry, Medical and Health Science Centre, University of Debrecen, Egyetem tér 1, H-4032 Debrecen, Hungary

E-mail: gpal@dote.hu

Key words: Zucker diabetic fatty rat, glycogen phosphorylase, glycogen synthase, glucose analogue inhibitors as cardiovascular disease, neuropathy, retinopathy and nephropathy. Type 2 diabetes comprises approximately $90-95 \%$ of all diabetes cases (1). Diabetes affects approximately 6-20\% of the adult population in Western industrialized societies (2) and is a major public health threat, reaching epidemic rates $(2,3)$. The disease is polygenic and is characterized by hyperglycemia, defects in insulin secretion and insulin resistance, in which the tissues fail to respond to this hormone (4). Additionally, the rate of endogenous glucose production is elevated in type 2 diabetes (5).

The liver accounts for $90 \%$ of the body's endogenous glucose production and is tightly controlled by insulin and glucagon. In type 2 diabetes, hepatic insulin resistance with elevated amounts of glucagon results in high levels of glucose, which in turn contributes to the observed hyperglycemia (6). Hepatic glucose is produced via two pathways: glycogenolysis (breakdown of glycogen) and gluconeogenesis (de novo synthesis of glucose). Studies estimate the glycogenolytic contribution of total hepatic glucose production to be up to $75 \%$ in both healthy controls (7) and patients with type 2 diabetes (8). Furthermore, a substantial portion of glucose formed by gluconeogenesis is cycled through the glycogen pool prior to efflux from the liver cells (9). The inhibition of either glycogenolysis or gluconeogenesis appears to be an attractive approach to developing antihyperglycemic molecules. Glycogen phosphorylase (GP), which catalyzes the first reaction in the degradation of glycogen, is considered a potential target for type 2 diabetes, generating selective ligands of GP that modulate the activity of this protein (10-15).

The accumulation and breakdown of glycogen are regulated by the reciprocal activities of glycogen synthase (GS) and GP. Only the phosphorylated GP $a$ has significant activity, suggesting an important role for glycogenolysis in hepatic glucose production. Therefore, the inhibition of hepatic GP might suppress glucose production arising from glycogenolysis and gluconeogenesis $(16,17)$. It is known that the activity of liver GS is also controlled by reversible phosphorylation of multiple serine residues. The dephosphorylated form of GS is the catalytically active (GSa) form. Phosphorylation is associated with the inactivation of GS: conversion to the $b$ form. The dephosphorylation of GP and GS is interrelated and is cata- 
lyzed by the glycogen-associated protein phosphatase-1. GP $a$, but not $\mathrm{GP} b$, is a potent inhibitor of the phosphatase action on GS, and it is only when GPa has been dephosphorylated that the phosphatase is free to activate GS, the rate limiting enzyme of glycogen synthesis $(2,18,19)$.

Rodents are commonly used for diabetic studies. Streptozotocin, a monofunctional nitrosourea derivative, is one of the most frequently applied substances to induce diabetes in experimental animals (20). Genetic models of insulin resistance and hyperglycemia are also available, including Zucker diabetic fatty (ZDF) rats. These rats have a mutation in the leptin receptor and a defect in the pancreatic $\beta$ cells that affect insulin production (21).

There are several binding sites in GP that lend themselves to targeting by various effectors. Many glucose derivatives were shown to bind to the catalytic centre of GP $(13,22)$, and among them glucopyranosylidene-spiro-hydantoin with a $K_{\mathrm{i}}$ of 3-4 $\mu \mathrm{M}$ was one of the most efficient inhibitors (23). More recent efforts resulted in the synthesis of a thio-analogue of the molecule with a very similar $K_{\mathrm{i}}$ value (24-26). In the present study, we report the effect of D-glucopyranosylidenespiro-thiohydantoin $(\mathrm{TH})$ on glycogen metabolism in the liver tissues of streptozotocin-induced and obese diabetic rats.

\section{Materials and methods}

Materials. TH was prepared from D-glucose in a six-step highly chemo-, regio- and stereoselective procedure (24-26). [ $\left.\gamma^{-32} \mathrm{P}\right]$ ATP (specific activity of $15 \mathrm{TBq} / \mathrm{mmol}$ ) was obtained from Izinta (Budapest, Hungary), and UDP- $\left[{ }^{14} \mathrm{C}\right]$-glucose was purchased from American Radiolabeled Chemicals (St. Louis, MO, USA). Sephadex G-25 columns $(1.5 \times 5 \mathrm{~cm})$ were supplied by Pharmacia (Uppsala, Sweden) and ET 31 filter paper by Whatman (Clifton, NJ, USA). Other reagents of analytical grade from various suppliers were used for the preparation of buffers.

Animals. Male Wistar rats weighing $250 \mathrm{~g}$ were obtained from Charles River Magyarország Kft. (Budapest, Hungary). Diabetes was induced by a simple intravenous injection of a freshly prepared solution of streptozotocin $(60 \mathrm{mg} / \mathrm{kg}$ body weight) dissolved in $50 \mathrm{mM}$ sodium citrate $(\mathrm{pH} 4.5)$ plus $150 \mathrm{mM} \mathrm{NaCl}$. Control rats were injected with citrate buffer alone. Diabetes was assessed periodically by a test for glucosuria (Macherey-Nagel, Düren, Germany). One week after diabetes induction, the animals were determined to be diabetic if urine glucose levels exceeded $3 \mathrm{mg} / \mathrm{ml}$. The diabetic animals were randomly divided into two groups: control diabetic and TH-treated diabetic. The control and the streptozotocininjected group were subcutaneously administered $0.9 \% \mathrm{NaCl}$ ( $2 \mathrm{ml} / \mathrm{kg}$ body weight) and the TH-treated streptozotocininjected group was subcutaneously administered $50 \mu \mathrm{M} \mathrm{TH}$ solution ( $100 \mu \mathrm{mol} / \mathrm{kg}$ body weight). TH was freshly dissolved in $0.9 \% \mathrm{NaCl}$ solution $(50 \mu \mathrm{mol} / \mathrm{ml})$. The treatments were performed 10 times between 8 and 9 a.m. every second day. Rats were anesthetized on the second day after the 10th treatment, the livers were removed by median laporotomy, and the wet liver weight was measured. ZDF/Gmi-fa/fa (ZDF) inbred obese male rats from Genetic Models, Inc. (Indianapolis, IN, USA) were ordered from Charles River Magyarország Kft.
All animals were cared for observing standard procedures with permission of the Laboratory Animals Protection Committee (29/2007/DE MÁB).

Gel filtered liver extract. The liver samples were immediately homogenized in a Potter-Elvehjem tube in two-volume ice-cold buffer consisting of $250 \mathrm{mM}$ sucrose and $40 \mathrm{mM}$ Tris- $\mathrm{HCl}$ ( $\mathrm{pH}$ 7.4), and centrifuged at $10,000 \mathrm{x} \mathrm{g}$ for $10 \mathrm{~min}$. The resulting post-mitochondrial supernatant was filtered through a Sephadex G-25 column $(5 \times 1.5 \mathrm{~cm})$ to remove endogenous effectors, equilibrated in $40 \mathrm{mM}$ Tris- $\mathrm{HCl}, 1 \mathrm{mM}$ EDTA and $10 \mathrm{mM}$ mercapto-ethanol ( $\mathrm{pH}$ 7.4). The filtrates were supplemented with $5 \mathrm{mM}\left(\mathrm{NH}_{4}\right)_{2} \mathrm{SO}_{4}$ and $1 \mathrm{mM}$ magnesium acetate in the presence of various effectors and incubated at $30^{\circ} \mathrm{C}$. Samples were withdrawn at the indicated times for the assay of GP or GS (27).

Assays. GP activity was assayed in the direction of glycogen synthesis at $30^{\circ} \mathrm{C}$ with $10 \mu \mathrm{g} / \mathrm{ml}$ enzyme and $1 \%$ glycogen in the absence of AMP (liver GPa) or in the presence of $2 \mathrm{mM}$ AMP (liver $\mathrm{GP} b$ ) in $50 \mathrm{mM}$ triethanolamine/ $\mathrm{HCl}(\mathrm{pH} 6.8)$ buffer, $100 \mathrm{mM} \mathrm{KCl}, 1 \mathrm{mM}$ dithiothreitol and $1 \mathrm{mM}$ EDTA. Hepatic GS was assayed according to Dopere et al in the absence or presence of $10 \mathrm{mM}$ glucose-6-phosphate (28). One unit of GP or GS converts $1 \mu \mathrm{mol}$ substrate into product per min at $30^{\circ} \mathrm{C}$. Protein was assayed as described by Bradford (29) and glycogen was measured using the phenol sulphuric acid assay (30). Glucose was determined by an enzymatic assay (Reanal, Hungary).

\section{Results}

Effect of TH on hepatic glycogen metabolism. It is known that glucose and glucose analogues cause a sequential inactivation (dephosphorylation) of liver GPa and activation (dephosphorylation) of GSb (31). The coordinated regulation of GP and GS was tested in gel-filtered rat liver extract. Fig. 1 illustrates the effect of $20 \mathrm{mM}$ glucose and $50 \mu \mathrm{M} \mathrm{TH}$ on the dephosphorylation of GPa in gel-filtered rat liver extract catalysed by phosphorylase phosphatase. Both ligands enhanced the dephosphorylation (inactivation) of hepatic $\mathrm{GP} a$; however, $\mathrm{TH}$ applied at a much lower concentration appeared to be more effective. Fig. 1 also illustrates the effect of either glucose or TH on the dephosphorylation (activation) of GS in gel-filtered rat liver extract. TH significantly increased the active form of GS in the liver by decreasing the latency in the dephosphorylation of GS.

Effect of TH on hepatic glycogen metabolism of streptozotocininduced rats. Streptozotocin is widely utilized to induce type 1 diabetes in rats with concomitant insulin deficiency (32), which is considered to be one of the animal models of human diabetes mellitus. One week after the injection of streptozotocin, the average blood glucose levels were $>30 \mathrm{mmol} / \mathrm{l}$ in the diabetic group, and remained at this level during the experimental period of 4 weeks (Fig. 2A). Hepatic glycogen content dramatically decreased in the diabetic animals from 24 to $2 \mathrm{mg} / \mathrm{g}$ in the liver. Diabetic animals treated with $100 \mu \mathrm{mol} / \mathrm{kg}$ body weight of TH exhibited significantly lower serum glucose levels, and the hepatic glycogen was also restored. 


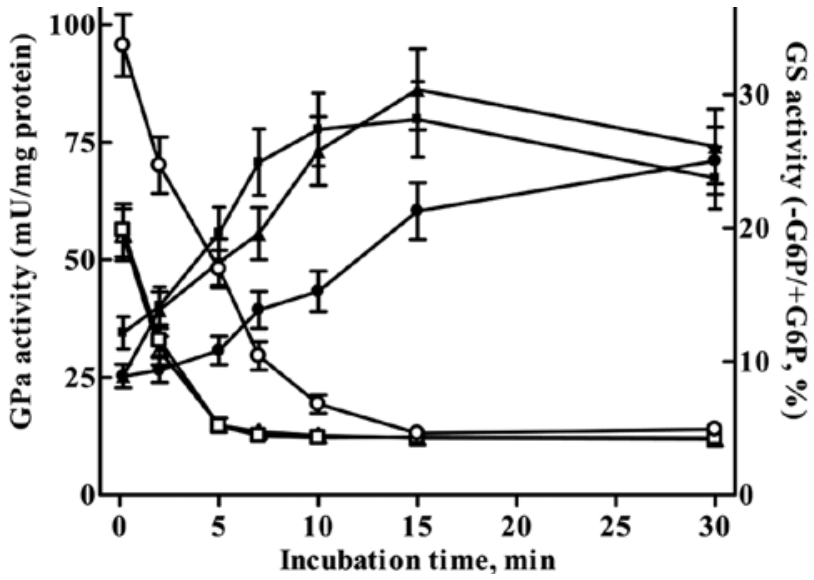

Figure 1. Effect of TH on the dephosphorylation of GPa and GS $b$ in gelfiltered liver extract of male Wistar rats. Post-mitochondrial supernatants were prepared and filtered through a Sephadex G-25 column, as described in Materials and methods. The filtrates were supplemented with $5 \mathrm{mM}$ $\left(\mathrm{NH}_{4}\right)_{2} \mathrm{SO}_{4}$ and $1 \mathrm{mM}$ magnesium acetate $(\mathrm{O}, \bullet)$ in the presence of $20 \mathrm{mM}$ glucose $(\square, \square)$ or $50 \mu \mathrm{M} \mathrm{TH}(\triangle, \mathbf{\Lambda})$, and incubated at $30^{\circ} \mathrm{C}$. Samples were withdrawn at the indicated times for the assay of GP $a$ or GS. The results are the mean $\pm \mathrm{SD}$ of four independent experiments.

The co-ordinated control of GP and GS in gel-filtered liver extracts in the three experimental groups is depicted in Fig. 2B. The diabetic group showed higher GPa activity compared to the control animals, while GS activity was markedly suppressed in the streptozotocin-treated rats. TH-treated diabetic rats had the lowest GP $a$ activity; however, GS activity was rather high, supporting the fact that glycogen concentration was elevated in the liver of this group.

Effect of TH on hepatic glycogen metabolism of Zucker diabetic fatty rats. The ZDF rat develops overt diabetes with severe hyperglycemia, polyurea and polydipsia. Therefore, it is a good type 2 diabetic model for testing small molecule antidiabetic compounds. As shown in Fig. 3, the co-ordinated control of GP and GS was observed in the gel-filtered extracts of spontaneously diabetic rats. When the GPa level was lowered, GS was sequentially dephosphorylated, and through this the activation of glycogen synthesis was initiated. Intravenous administration of TH significantly decreased the GP $a$ level and the activation of GS was initiated without any delay.

\section{Discussion}

Liver GP has been identified as a novel target for drugs that control blood glucose concentration. Several compounds with different chemical structures have been probed for the inhibition of hepatic GP. Glucose and its analogues were shown to be inhibitors of the enzyme. TH was found to be a potent glucose derivative that inhibits the catalytic activity of both muscle and liver GP enzymes $(24,25)$. By means of a synthetic procedure using stereoselective preparation, it is possible to produce gram quantities of TH in six-to-seven steps starting from D-glucose $(25,26)$. The structure of the $\mathrm{GP} b$-TH complex as revealed by X-ray studies demonstrates that the inhibitor can be accomodated in the catalytic site of T-state GP $b$ with very little change in the tertiary structure, and provides a basis for understanding the potency and specificity of TH (33).
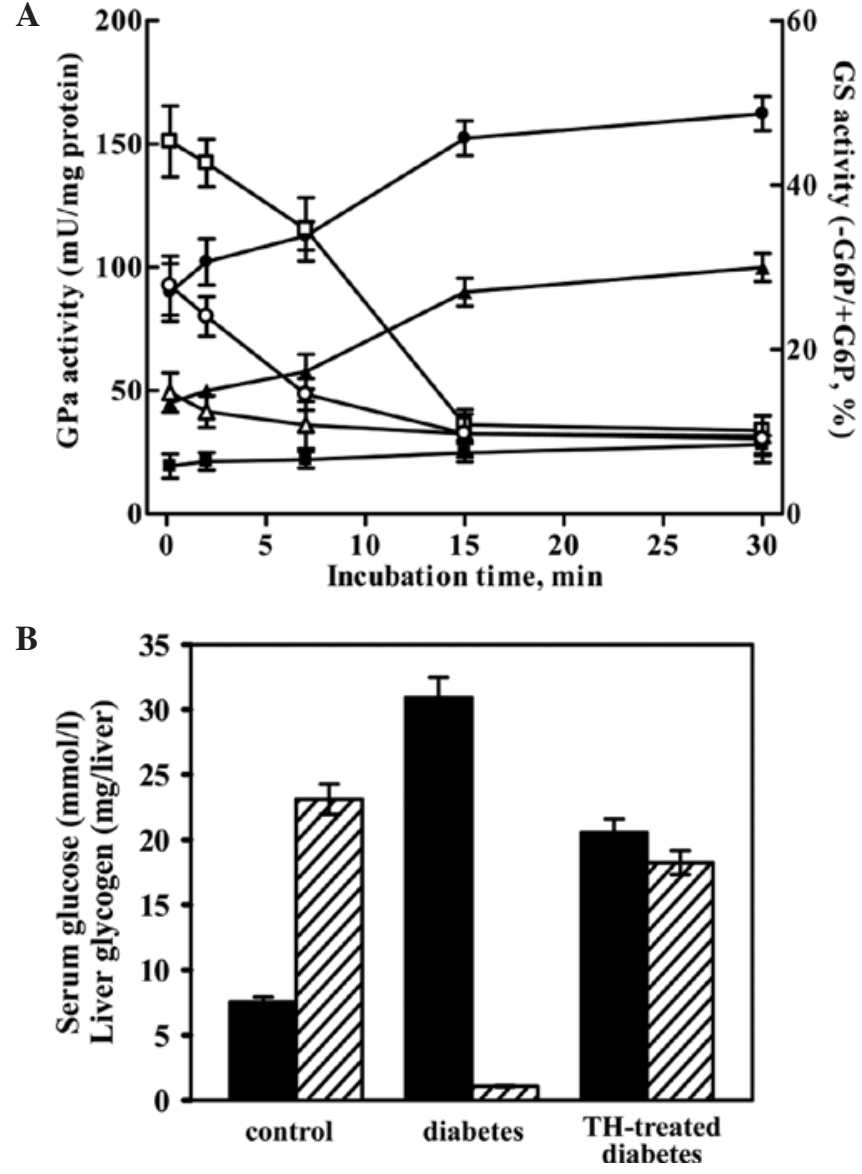

Figure 2. Serum glucose, hepatic glycogen, GP and GS levels in streptozotocininduced diabetic rats treated with TH. (A) Inactivation of GP (empty symbols) and activation of GS (filled symbols) in gel-filtered liver extracts. Postmitochondrial supernatants were prepared and filtered through a Sephadex G-25 column, as described in Materials and methods. The filtrates were incubated at $30^{\circ} \mathrm{C}$ and samples were withdrawn at the indicated times for the assay of GP $a$ or GS. Control $(\bigcirc, \bullet)$, diabetic control $(\square, \boldsymbol{\bullet})$ and TH-treated diabetic rats $(\triangle, \mathbf{\Lambda})$. (B) Effect of TH treatment on the concentration of blood glucose (filled column) and liver glycogen (hatched column) in streptozotocin-induced diabetic rats. The results are the mean $\pm \mathrm{SD}$ of four independent experiments.

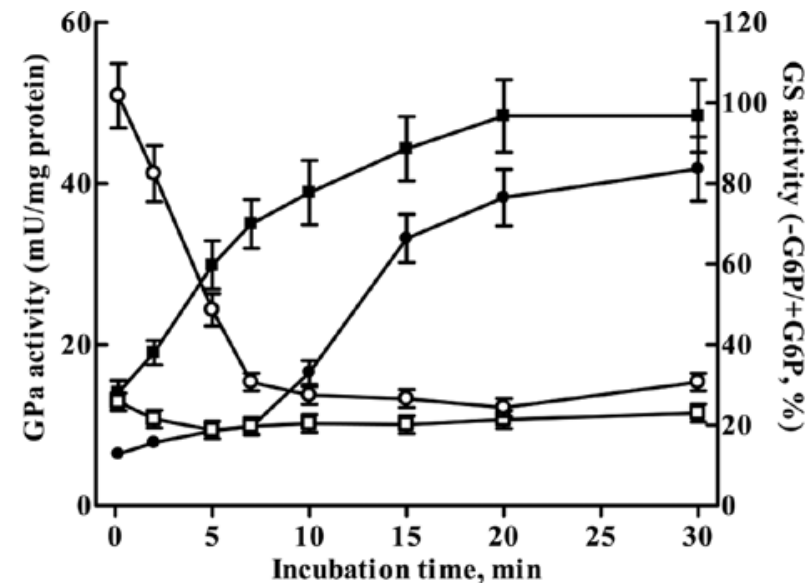

Figure 3. Effect of intravenously injected TH on the inactivation of GPa and activation of GS $b$ in gel-filtered rat liver extract of spontaneously diabetic Zucker rats. Liver samples were removed from different liver lobules and post-mitochondrial supernatants were prepared and filtered through a Sephadex G-25 column, as described in Materials and methods. Control biopsy $(\bigcirc, \bullet)$; after the intravenous injection of $\mathrm{TH}(100 \mu \mathrm{mol} / \mathrm{kg}$ body weight) to the portal vein at $5 \mathrm{~min}(\square, \boldsymbol{\square})$. The results are the mean $\pm \mathrm{SD}$ of four independent experiments. 
Glucose and its analogues cause a sequential inactivation of GPa and activation (dephosphorylation) of GS $b$ in the liver. We examined the co-ordinated regulation of GP and GS in rat liver extract and observed that $50 \mu \mathrm{M}$ TH promoted the activation of GS by significantly decreasing the latency in the dephosphorylation of GS. The decrease of GPa activity, the increase of phosphatase activity and the increase of GS activity all result in the same biological response: the inhibition of hepatic glycogen breakdown, which in turn decreases the concentration of blood glucose. The mechanism by which TH inactivates GPa and activates GS may be complex and requires further study. However, the observed effects of $\mathrm{TH}$ clearly indicate that this compound and its analogues may be of potential use in controlling hyperglycaemia.

It is known that streptozotocin-induced diabetes influences glycogen metabolism in animal models. Diabetic rats showed an approximately 4-fold higher blood glucose concentration and approximately 3 -fold decrease in liver glycogen concentration compared to control or insulintreated diabetic animals (34). Similar results are depicted in Fig. 2. Furthermore, TH treatment restored these values to physiological ranges. Our results suggest that the administration of $\mathrm{TH}$ improves hyperglycemia, while inhibiting the breakdown and activating the synthesis of glycogen. The $\mathrm{ZDF}$ rats also responded to the $\mathrm{TH}$ treatment; the active form of GP was decreased, which in turn triggered the synthesis of glycogen by activating GS (Fig. 3). FR258900, a GP inhibitor isolated from the cultured broth of a fungal strain, also exhibited hypoglycaemic effects in diabetic animal models. FR258900 treatment significantly reduced plasma glucose concentrations during oral glucose tolerance tests in diabetic mice models, including $d b / d b$ mice and streptozotocin-induced diabetic mice (35).

It would appear that specific inhibition of hepatic GP results in a rapid decrease in plasma glucose levels in diabetic animals. These improvements in glucose disposal are accompanied by increased liver glycogen contents, suggesting that the glucose-lowering effect was the result of suppressed hepatic glycogen breakdown and increased hepatic glycogen synthesis. Taken together, our results suggest that GP is a potentially useful target in novel therapies against diabetes. The design and development of further glucose analogues for GP as potential therapeutic targets for diabetes have been comprehensively reviewed $(13,14,22,36)$. These compounds primarily bind to the active site of GP, characterized by a D-glucopyranose ring having either diverse substituents or spirocyclic moieties attached to the anomeric carbon. Exhaustive survey of non-glucose-type inhibitors targeting binding sites of GP other than the catalytic site has been conducted $(13,14,37)$. The latest findings from physiological studies on novel GP inhibitors have been summarized by Agius (15).

\section{Acknowledgements}

This study was supported by grants from the Hungarian Science Research Fund (OTKA CNK80709, CK77712) and the TÁMOP 4.2.1./B-09/1/KONV-2010-0007 project implemented through the New Hungary Development Plan, co-financed by the European Social Fund.

\section{References}

1. Colberg SR: Enhancing insulin action with physical activity to prevent and control diabetes. ACSM'S Health and Fitness Journal 12: 16-22, 2008

2. Zimmet P, Alberti KG and Shaw J: Global and societal implications of the diabetes epidemic. Nature 414: 782-787, 2001.

3. Dai YD, Marrero IG, Gros P, Zaghouani H, Wicker LS and Sercarz EE: Slc1la1 enhances the autoimmune diabetogenic T-cell response by altering processing and presentation of pancreatic islet antigens. Diabetes 58: 156-164, 2009.

4. Henke BR and Sparks SM: Glycogen phosphorylase inhibitors. Mini Rev Med Chem 6: 845-857, 2006.

5. Roden $\mathrm{M}$ and Bernroider E: Hepatic glucose metabolism in humans - its role in health and disease. Best Pract Res Clin Endocrinol Metab 17: 365-383, 2003.

6. Choi K and Kim YB: Molecular mechanism of insulin resistance in obesity and type 2 diabetes. Korean J Intern Med 25: 119-129, 2010.

7. Magnusson I, Rothman DL, Gerard DP, Katz LD and Shulman GI: Contribution of hepatic glycogenolysis to glucose production in humans in response to a physiological increase in plasma glucagon concentration. Diabetes 44: 185-189, 1995.

8. Diraison F, Large V, Brunengraber $\mathrm{H}$ and Beylot M: Non-invasive tracing of liver intermediary metabolism in normal subjects and in moderately hyperglycaemic NIDDM subjects. Evidence against increased gluconeogenesis and hepatic fatty acid oxidation in NIDDM. Diabetologia 41: 212-220, 1998.

9. Andersen B, Rassov A, Westergaard $\mathrm{N}$ and Lundgren $\mathrm{K}$ : Inhibition of glycogenolysis in primary rat hepatocytes by 1, 4-dideoxy-1,4-imino-D-arabinitol. Biochem J 342 (Pt 3): 545-550, 1999.

10. Treadway JL, Mendys P and Hoover DJ: Glycogen phosphorylase inhibitors for treatment of type 2 diabetes mellitus. Expert Opin Investig Drugs 10: 439-454, 2001.

11. Somsak L, Nagya V, Hadady Z, Docsa T and Gergely P: Glucose analog inhibitors of glycogen phosphorylases as potential antidiabetic agents: recent developments. Curr Pharm Des 9: 1177-1189, 2003.

12. Czifrak K, Kovacs L, Kover KE and Somsak L: Synthesis of some derivatives of C-(1-deoxy-1-N-substituted-D-glucopyranosyl) formic acid (D-gluco-hept-2-ulopyranosonic acid) as potential inhibitors of glycogen phosphorylase. Carbohydr Res 340: 2328-2334, 2005 .

13. Somsak L, Czifrak K, Toth M, et al: New inhibitors of glycogen phosphorylase as potential antidiabetic agents. Curr Med Chem 15: 2933-2983, 2008.

14. Oikonomakos NG and Somsak L: Advances in glycogen phosphorylase inhibitor design. Curr Opin Investig Drugs 9: 379-395, 2008.

15. Agius L: Physiological control of liver glycogen metabolism: lessons from novel glycogen phosphorylase inhibitors. Mini Rev Med Chem 10: 1175-1187, 2010.

16. Gerich JE: Control of glycaemia. Baillieres Clin Endocrinol Metab 7: 551-586, 1993

17. Gerich JE and Nurjhan N: Gluconeogenesis in type 2 diabetes. Adv Exp Med Biol 334: 253-258, 1993.

18. Tung HY, Alemany S and Cohen P: The protein phosphatases involved in cellular regulation. 2. Purification, subunit structure and properties of protein phosphatases-2A0, $2 \mathrm{~A} 1$, and $2 \mathrm{~A} 2$ from rabbit skeletal muscle. Eur J Biochem 148: 253-263, 1985.

19. Somsak L, Kovacs L, Toth M, et al: Synthesis of and a comparative study on the inhibition of muscle and liver glycogen phosphorylases by epimeric pairs of d-gluco- and d-xylopyranosylidene-spiro-(thio)hydantoins and N-(d-glucopyranosyl) amides. J Med Chem 44: 2843-2848, 2001.

20. Szkudelski T: The mechanism of alloxan and streptozotocin action in B cells of the rat pancreas. Physiol Res 50: 537-546, 2001.

21. Griffen SC, Wang J and German MS: A genetic defect in beta-cell gene expression segregates independently from the fa locus in the ZDF rat. Diabetes 50: 63-68, 2001.

22. Praly JP and Vidal S: Inhibition of glycogen phosphorylase in the context of type 2 diabetes, with focus on recent inhibitors bound at the active site. Mini Rev Med Chem 10: 1102-1126, 2010.

23. Bichard CJF, Mitchell EP, Wormald MR, et al: Potent inhibition of glycogen-phosphorylase by a spirohydantoin of glucopyranose - first pyranose analogs of hydantocidin. Tetrahedron Letters 36: 2145-2148, 1995. 
24. Osz E, Somsak L, Szilagyi L, et al: Efficient inhibition of muscle and liver glycogen phosphorylases by a new glucopyranosylidenespiro-thiohydantoin. Bioorg Med Chem Lett 9: 1385-1390, 1999.

25. Somsak L, Nagy V, Docsa T, Toth B and Gergely P: Gram-scale synthesis of a glucopyranosylidene-spiro-thiohydantoin and its effect on hepatic glycogen metabolism studied in vitro and in vivo. Tetrahedron-Asymmetry 11: 405-408, 2000.

26. Somsak L and Nagy V: A new, scalable preparation of a glucopyranosylidene-spiro-thiohydantoin: one of the best inhibitors of glycogen phosphorylases. Tetrahedron Asymmetry 11: $1719-1727,2000$

27. Farkas I, Toth B, Bot G and Gergely P: Hormonal regulation of phosphorylase phosphatase activity in rat liver. FEBS Lett 203 : 253-256, 1986.

28. Dopere F, Goris J, Vandenheede JR and Merlevede W: Cyclic AMP- and $\mathrm{Ca} 2+-$-independent glycogen synthase kinase from dog liver. Biochem Soc Trans 8: 526-527, 1980.

29. Bradford MM: A rapid and sensitive method for the quantitation of microgram quantities of protein utilizing the principle of protein-dye binding. Anal Biochem 72: 248-254, 1976.

30. Lo S, Russell JC and Taylor AW: Determination of glycogen in small tissue samples. J Appl Physiol 28: 234-236, 1970.
31. Stalmans W, Bollen M and Mvumbi L: Control of glycogen synthesis in health and disease. Diabetes Metab Rev 3: 127-161, 1987.

32. Bolzan AD and Bianchi MS: Genotoxicity of streptozotocin. Mutat Res 512: 121-134, 2002.

33. Oikonomakos NG, Skamnaki VT, Osz E, et al: Kinetic and crystallographic studies of glucopyranosylidene spirothiohydantoin binding to glycogen phosphorylase B. Bioorg Med Chem 10: 261-268, 2002.

34. Khandelwal RL, Zinman SM and Zebrowski EJ: The effect of streptozotocin-induced diabetes and of insulin supplementation on glycogen metabolism in rat liver. Biochem J 168: 541-548, 1977.

35. Furukawa S, Murakami K, Nishikawa M, Nakayama O and Hino M: FR258900, a novel glycogen phosphorylase inhibitor isolated from Fungus No. 138354. II. Anti-hyperglycemic effects in diabetic animal models. J Antibiot 58: 503-506, 2005.

36. Gimisis T: Synthesis of N-glucopyranosidic derivatives as potential inhibitors that bind at the catalytic site of glycogen phosphorylase. Mini Rev Med Chem 10: 1127-1138, 2010.

37. Loughlin WA: Recent advances in the allosteric inhibition of glycogen phosphorylase. Mini Rev Med Chem 10: 1139-1155, 2010 . 